Pacific

Journal of

Mathematics

\title{
ENERGY AND TOPOLOGY OF SINGULAR UNIT VECTOR FIELDS ON $S^{3}$
}

\author{
Pablo M. Chacón and Giovanni Da Silva Nunes
}




\title{
ENERGY AND TOPOLOGY OF SINGULAR UNIT VECTOR FIELDS ON $S^{3}$
}

\author{
Pablo M. Chacón and Giovanni da Silva Nunes
}

\begin{abstract}
The energy of unit vector fields on odd-dimensional spheres is a functional that has a minimum in dimension 3 and an infimum in higher dimensions. Vector fields with isolated singularities arise naturally in the study of this functional. We consider the class of fields in $S^{\mathbf{3}}$ having two antipodal singularities. We prove a lower bound, attained for the radial vector field, for the energy of this class of fields in terms of the indices of the singularities. A similar inequality is not to be expected in other dimensions.
\end{abstract}

\section{Introduction}

Let $\left(M^{n+1}, g\right)$ be a compact oriented Riemannian manifold without boundary. We denote by $\nabla$ the Levi-Civita connection and by $v$ the volume form. Given a unit vector field $\vec{v}$ on $M$, the energy of $\vec{v}$ is (see [Wiegmink 1995])

$$
\mathscr{E}(\vec{v})=\frac{1}{2} \int_{M}\|\nabla \vec{v}\|^{2} v+\frac{1}{2}(n+1) \operatorname{vol} M .
$$

This integral (times a constant) is also known as the total bending of $\vec{v}$ or the vertical energy of $\vec{v}$ as a section of the tangent bundle. The integrand $\frac{1}{2}\|\nabla \vec{v}\|^{2}$ is called the energy density of $\vec{v}$.

The absolute minimum of the energy functional is $\frac{1}{2}(n+1)$ vol $M$, attained for vector fields whose integral curves are geodesic and such that the orthogonal distribution $\vec{v}^{\perp}$ is integrable and totally geodesic. Such vector fields, called parallel, are rare because if $M$ admits a unit parallel vector field then $M$ is locally a Riemannian product.

The simplest spaces to be studied are spheres.

Theorem 1 [Brito 2000]. Hopf vector fields are the only unit vector fields on $\mathbf{S}^{3}$ to minimize $\mathscr{E}$.

MSC2000: primary 53C20; secondary 57R25, 58C25.

Keywords: energy of vector field, index of vector field.

Chacón is partially supported by MEC/FEDER Grant No. MTM2004-04934-C04-02 (Spain). This work was initiated during a postdoctoral stay of his funded by DGU No. HBE2002-008 (Spain). 
By a Hopf vector field we mean a unit vector field tangent to the classical Hopf fibration. In dimensions 5 and higher, Hopf vector fields are unstable critical points of $\mathscr{E}$; see [Wood 2000; Gil-Medrano and Llinares-Fuster 2001].

The radial vector field $V_{R}$ plays a fundamental role in the study of the energy. It is defined, relative to fixed antipodal points $N, S \in \mathbf{S}^{n+1}$, as the unit vector field tangent to the geodesics from $N$ to $S$. It has $\{N, S\}$ as its only singularities.

Theorem 2 [Brito and Walczak 2000]. The energy of any unit vector field $\vec{v}$ with isolated singularities on $\mathbf{S}^{n+1}, n \geq 2$, satisfies the inequality

$$
\mathscr{E}(\vec{v}) \geq \frac{n^{2}+n-1}{2(n-1)} \operatorname{vol} \mathbf{S}^{n+1},
$$

and if $n \geq 3$, equality holds if and only if $\vec{v}=V_{R}$.

The existence of a global energy-minimizing unit vector field was left open at that time. This question has since been solved:

Theorem 3 [Borrelli et al. 2003]. The infimum of $\mathscr{E}$ among all smooth, globally defined unit vector fields on sphere $\mathbf{S}^{2 k+1}$, for $k \geq 2$, is

$$
\frac{4 k^{2}+2 k-1}{2(2 k-1)} \operatorname{vol} \mathbf{S}^{2 k+1} \text {. }
$$

In fact, the proof of Theorem 3 in [Borrelli et al. 2003] shows a family of unit vector fields whose energy converges to $\mathscr{E}\left(V_{R}\right)$.

More results on energy can be found, for example, in [Berndt et al. 2003; Boeckx et al. 2002; Brito and Salvai 2004; Chacón and Naveira 2004; Gil-Medrano et al. 2004; Gil-Medrano and Hurtado 2004; González-Dávila and Vanhecke 2002; Wiegmink 1996].

From Theorem 1 and 3 it follows that the energy on $\mathbf{S}^{2 k+1}$ has a minimum only in dimension 3. It is easy to see that on $\mathbf{S}^{3}$ Hopf vector fields and radial vector fields have the same energy. Thus vector fields with singularities appear naturally in the study of the energy of global unit vector fields.

In this paper we relate the energy and topology of vector fields with singularities. More precisely, we prove:

Theorem 4. Let $\vec{v}$ be a unit vector field on $\mathbf{S}^{3}$ with exactly two antipodal singularities of index $\pm k$ with $k \in \mathbb{N}$. Then

$$
\mathscr{E}(\vec{v}) \geq\left(|2 k-1|+\frac{3}{2}\right) \operatorname{vol}\left(\mathbf{S}^{3}\right) .
$$

The radial vector field $V_{R}$ satisfies the equality in Theorem 4 . The indices of $V_{R}$ at $N$ and $S$ are respectively +1 and -1 . For vector fields with indices \pm 2 , we do not know if there is a vector field for which equality obtains in Theorem 4. 
A similar result is not expected for higher-dimensional spheres. The energy density of $\vec{v}$ has degree 2 . The index of the singularity of a vector field on $\mathbf{S}^{n+1}$ is defined through a map from $\mathbf{S}^{n}$ to $\mathbf{S}^{n}$. The underlying technique in proving Theorem 4 is to relate the energy density (of degree 2) to an integral of a $n$-form of $\mathbf{S}^{n}$ that gives the index. For $n=2$, all these objects are comparable but not for higher dimensions; the $n$-form of the index will not provide the energy density.

\section{Notation and definitions}

On $\mathbf{S}^{3}$, take two antipodal points $\{N, S\}$, determining the northern and southern hemispheres. Consider a vector field $\vec{v}$ on $\mathbf{S}^{3}$ such that $\vec{v}(N)=\vec{v}(S)=0$ and $\|\vec{v}(p)\|=1$ for $p \notin\{N, S\}$. The energy of $\vec{v}$ is defined in (1).

We fix an orientation for $\mathbf{S}^{3}$ and choose an oriented orthonormal local frame $\left\{e_{1}, e_{2}, e_{3}\right\}$ on $\mathbf{S}^{3} \backslash\{N, S\}$ such that $e_{3}=\vec{v}$. The corresponding dual 1-forms are denoted by $\left\{\theta_{1}, \theta_{2}, \theta_{3}\right\}$. The connection forms of the Levi-Civita connection $\nabla$ of $\mathbf{S}^{3}$ will be denoted by $\omega_{i j}$ :

$$
\omega_{i j}(X)=g\left(\nabla_{X} e_{i}, e_{j}\right) \quad \text { for } i, j=1,2,3,
$$

where $X$ is a vector in the corresponding tangent space.

On $\mathbf{S}^{3} \backslash\{N, S\}$ the field $\vec{v}$ determines a subbundle $\vec{v}^{\perp}$ of $T \mathbf{S}^{3}$ formed by the vectors orthogonal to $\vec{v}$. The second fundamental form of $\vec{v}^{\perp}$, possibly nonsymmetric, is given by the coefficients $h_{i j}=\omega_{j 3}\left(e_{i}\right)$ for $i, j=1,2$. The energy density of $\vec{v}$ is

$$
\frac{1}{2}\|\nabla \vec{v}\|^{2}=\frac{1}{2}\left(\sum_{i, j=1}^{2} h_{i j}^{2}+\sum_{i=1}^{2} a_{i}^{2}\right),
$$

where the $a_{i}$ are the components of the acceleration of $\vec{v}$ :

$$
\nabla_{e_{3}} \vec{v}=\nabla_{\vec{v}} \vec{v}=a_{1} e_{1}+a_{2} e_{2} .
$$

\section{Proof of Theorem 4}

Since $\mathbf{S}^{3}$ has Euler characteristic zero, the sum of the indices of $\vec{v}$ at the singularities must be zero. If the singularities of $\vec{v}$ have index zero, Theorem 4 follows from Theorem 2. We may therefore assume that $\vec{v}$ has nontrivial singularities.

We may also assume that $N$ is the singularity with index $+k$ and $S$ is the singularity with index $-k$. For simplicity, we also assume that $N=(0,0,0,1) \in \mathbb{R}^{4}$ and $S=(0,0,0,-1) \in \mathbb{R}^{4}$.

Consider the 2-form on $\mathbf{S}^{3} \backslash\{N, S\}$ given by $\omega_{13} \wedge \omega_{23}$. It is not hard to see that

$$
\begin{aligned}
\omega_{13} \wedge \omega_{23}=\left(h_{11} h_{22}-\right. & \left.h_{12} h_{21}\right) \theta_{1} \wedge \theta_{2} \\
& +\left(h_{12} a_{1}-h_{11} a_{2}\right) \theta_{1} \wedge \theta_{3}+\left(h_{22} a_{1}-h_{21} a_{2}\right) \theta_{2} \wedge \theta_{3} .
\end{aligned}
$$


This form is independent of the oriented frame chosen (satisfying $e_{3}=\vec{v}$ ). Let

$$
S_{\theta}=\left\{(x, y, z, t) \in \mathbf{S}^{3} \mid t=\sin \theta\right\} \equiv \mathbf{S}^{2}(\cos \theta)
$$

be the "parallel of latitude" in $\mathbf{S}^{3}$ determined by $\theta \in(-\pi / 2, \pi / 2)$. The field $\vec{v}$ has no singularities on any $S_{\theta}$. Let $\alpha \in[0, \pi / 2]$ be the angle between $\vec{v}$ and $T S_{\theta}$. Note that $\cos \alpha$ and $\sin \alpha$ are well-defined functions on $S_{\theta}$. Take an oriented orthonormal local frame $\left\{e_{1}, e_{2}, \vec{v}\right\}$ such that $e_{1} \in T S_{\theta}$. We choose the orientation on the parallel $S_{\theta}$ given by the normal vector field $V_{R}$ (pointing downward). Depending on the index of the singularity, along a fix parallel $S_{\theta}$ near the singularity, the field $\vec{v}$ can cross the plane $T S_{\theta}$ several times, or equivalently $V_{R}$ and $\vec{v}$ can be on the same or on different sides of $T \mathbf{S}^{3}$ in relation with $T S_{\theta}$. We define $\epsilon_{1}=-1$ if $T S_{\theta}$ leaves $V_{R}$ and $\vec{v}$ on different sides and $\epsilon_{1}=1$ otherwise. Next set

$$
\tilde{e}=\sin \alpha e_{2}+\epsilon_{2} \cos \alpha \vec{v}
$$

where $\epsilon_{2}=1$ if $T S_{\theta}$ separates $e_{2}$ and $\vec{v}$ on different sides of $T \mathbf{S}^{3}$ and $\epsilon_{2}=-1$ otherwise. Then $\left\{e_{1}, \epsilon_{1} \tilde{e}\right\}$ is an oriented local frame of $S_{\theta}$. When $e_{2} \in T S_{\theta}$, we have $\cos \alpha=0$ and the value of $\epsilon_{2}$ is irrelevant. For the special case $\vec{v} \in T S_{\theta}$, we have $\sin \alpha=0$, and the oriented frame of $S_{\theta}$ is $\left\{e_{1},-\vec{v}\right\}$ when $e_{2}$ agrees with $V_{R}$ or $\left\{e_{1}, \vec{v}\right\}$ when $e_{2}=-V_{R}$. Observe that although the positions of $\vec{v}, V_{R}, T S_{\theta}$ and $e_{2}$ can change, the frame $\left\{e_{1}, \epsilon_{1} \tilde{e}\right\}$ is a $C^{\infty}$ local frame of $S_{\theta}$ (not global but differentiable).

If $i: S_{\theta} \rightarrow \mathbf{S}^{3}$ is the inclusion map, we have the 2 -form $i^{*}\left(\omega_{13} \wedge \omega_{23}\right)$ on $S_{\theta}$. Using (2), we can evaluate

$$
\begin{aligned}
i^{*}\left(\omega_{13} \wedge \omega_{23}\right)\left(e_{1}, \epsilon_{1} \tilde{e}\right) & =\epsilon_{1} \omega_{13} \wedge \omega_{23}\left(e_{1}, \sin (\alpha) e_{2}+\epsilon_{2} \cos (\alpha) \vec{v}\right) \\
& =\epsilon_{1} \sin \alpha\left(h_{11} h_{22}-h_{12} h_{21}\right)+\epsilon_{1} \epsilon_{2} \cos \alpha\left(h_{12} a_{1}-h_{11} a_{2}\right) .
\end{aligned}
$$

This value depends only on $\vec{v}$.

We next compute

$$
\begin{aligned}
& \sum_{i, j=1}^{2} h_{i j}^{2}+\sum_{i=1}^{2} a_{i}^{2} \\
&=(\sin \alpha)^{2} h_{11}^{2}+h_{22}^{2}+(\sin \alpha)^{2} h_{21}^{2}+h_{12}^{2} \\
& \quad+\left(\epsilon_{2} \cos \alpha\right)^{2} h_{21}^{2}+a_{1}^{2}+\left(\epsilon_{2} \cos \alpha\right)^{2} h_{11}^{2}+a_{2}^{2} \\
& \geq 2\left|\sin \alpha h_{11} h_{22}\right|+2\left|\sin \alpha h_{21} h_{12}\right|+2\left|\epsilon_{2} \cos \alpha h_{21} a_{1}\right|+2\left|\epsilon_{2} \cos \alpha h_{11} a_{2}\right| \\
& \geq 2\left|\sin \alpha\left(h_{11} h_{22}-h_{12} h_{21}\right)\right|+2\left|\epsilon_{2} \cos \alpha\left(h_{21} a_{1}-h_{11} a_{2}\right)\right| \\
& \geq 2\left|\sin \alpha\left(h_{11} h_{22}-h_{12} h_{21}\right)+\epsilon_{2} \cos \alpha\left(h_{12} a_{1}-h_{11} a_{2}\right)\right| \\
&=2\left|i^{*}\left(\omega_{13} \wedge \omega_{23}\right)\left(e_{1}, \tilde{e}\right)\right|=2\left|i^{*}\left(\omega_{13} \wedge \omega_{23}\right)\left(e_{1}, \epsilon_{1} \tilde{e}\right)\right|
\end{aligned}
$$


(recall that $x^{2}+y^{2} \geq 2|x y|$ for $x, y \in \mathbb{R}$ ). In this way, we can bound the integral in (1) from below by

$$
\begin{aligned}
\int_{\mathbf{S}^{3}}\|\nabla \vec{v}\|^{2} v & =\int_{\mathbf{S}^{3}}\left(\sum_{i, j=1}^{2} h_{i j}^{2}+\sum_{i=1}^{2} a_{i}^{2}\right) v \geq \int_{-\pi / 2}^{\pi / 2} \int_{S_{\theta}} 2\left|i^{*}\left(\omega_{13} \wedge \omega_{23}\right)\left(e_{1}, \epsilon_{1} \tilde{e}\right)\right| v \\
= & \int_{0}^{\pi / 2} \int_{S_{\theta}} 2\left|i^{*}\left(\omega_{13} \wedge \omega_{23}\right)\left(e_{1}, \epsilon_{1} \tilde{e}\right)\right| v_{\theta} d \theta \\
& +\int_{-\pi / 2}^{0} \int_{S_{\theta}} 2\left|i^{*}\left(\omega_{13} \wedge \omega_{23}\right)\left(e_{1}, \epsilon_{1} \tilde{e}\right)\right| v_{\theta} d \theta
\end{aligned}
$$

where $v_{\theta}$ is the volume form of $S_{\theta}$. Therefore,

$$
\begin{aligned}
\int_{\mathbf{S}^{3}}\|\nabla \vec{v}\|^{2} v \geq \mid 2 \int_{0}^{\pi / 2} \int_{S_{\theta}} i^{*}\left(\omega_{13}\right. & \left.\wedge \omega_{23}\right)\left(e_{1}, \epsilon_{1} \tilde{e}\right) v_{\theta} d \theta \mid \\
+ & \left|2 \int_{-\pi / 2}^{0} \int_{S_{\theta}} i^{*}\left(\omega_{13} \wedge \omega_{23}\right)\left(e_{1}, \epsilon_{1} \tilde{e}\right) v_{\theta} d \theta\right|
\end{aligned}
$$

The integral over $(0, \pi / 2)$ in (4) is related to the index of $\vec{v}$ at $N$ and the integral over $(-\pi / 2,0)$ is related to the index of $\vec{v}$ at $S$. We consider the first integral.

By the structure equations of $\mathbf{S}^{3}$, the form $\omega_{13} \wedge \omega_{23}+\theta_{1} \wedge \theta_{2}$ is closed and hence

$$
d\left(\omega_{13} \wedge \omega_{23}\right)=-d\left(\theta_{1} \wedge \theta_{2}\right) .
$$

To integrate the first term of (4), we consider the region of $\mathbf{S}^{3}$ defined by

$$
A_{\phi \psi}=\left\{(x, y, z, t) \in \mathbf{S}^{3} \mid \sin \phi \leq t \leq \sin \psi\right\},
$$

where $0 \leq \phi<\psi \leq \pi / 2$.

By Stokes' Theorem and the appropriate orientation of $A_{\phi \psi}$ we know that

$$
\int_{A_{\phi \psi}} d\left(\omega_{13} \wedge \omega_{23}\right)=\int_{S_{\psi}} i^{*}\left(\omega_{13} \wedge \omega_{23}\right)-\int_{S_{\phi}} i^{*}\left(\omega_{13} \wedge \omega_{23}\right) .
$$

On the other hand, by (5) and Stokes' Theorem we have

$$
\int_{A_{\phi \psi}} d\left(\omega_{13} \wedge \omega_{23}\right)=-\int_{A_{\phi \psi}} d\left(\theta_{1} \wedge \theta_{2}\right)=\int_{S_{\phi}} i^{*}\left(\theta_{1} \wedge \theta_{2}\right)-\int_{S_{\psi}} i^{*}\left(\theta_{1} \wedge \theta_{2}\right)
$$

We can calculate the value of $i^{*}\left(\theta_{1} \wedge \theta_{2}\right)$ :

$$
i^{*}\left(\theta_{1} \wedge \theta_{2}\right)\left(e_{1}, \epsilon_{1} \tilde{e}\right)=\epsilon_{1}\left(\theta_{1} \wedge \theta_{2}\right)\left(e_{1}, \sin (\alpha) e_{2}+\epsilon_{2} \cos (\alpha) \vec{v}\right)=\epsilon_{1} \sin \alpha .
$$


So, for any $\phi, 0 \leq \phi<\pi / 2$, by (6), (7) and (8) we obtain

$$
\begin{aligned}
\int_{S_{\phi}} i^{*}\left(\omega_{13} \wedge \omega_{23}\right) & =\int_{S_{\psi}} i^{*}\left(\omega_{13} \wedge \omega_{23}\right)+\int_{S_{\psi}} i^{*}\left(\theta_{1} \wedge \theta_{2}\right)-\int_{S_{\phi}} i^{*}\left(\theta_{1} \wedge \theta_{2}\right) \\
& =\int_{S_{\psi}} i^{*}\left(\omega_{13} \wedge \omega_{23}\right)+\int_{S_{\psi}} \epsilon_{1} \sin \alpha v_{\psi}-\int_{S_{\phi}} \epsilon_{1} \sin \alpha v_{\phi} \\
& \geq \int_{S_{\psi}} i^{*}\left(\omega_{13} \wedge \omega_{23}\right)-\operatorname{vol} S_{\psi}-\operatorname{vol} S_{\phi} .
\end{aligned}
$$

From the definition of the index and the fact that the degree of a map $f: S_{\psi} \rightarrow \mathbf{S}^{2}$ is equal to $\left(\operatorname{vol} \mathbf{S}^{2}\right)^{-1} \int_{S_{\psi}} f^{*} \eta$, where $\eta$ is a volume form of $\mathbf{S}^{2}$, we obtain an integral expression for the index of $\vec{v}$ at the singularity $N$ :

$$
\lim _{\psi \rightarrow \frac{\pi}{2}} \int_{S_{\psi}} i^{*}\left(\omega_{13} \wedge \omega_{23}\right)=4 \pi k
$$

From (9) and (10) we obtain $\int_{S_{\phi}} i^{*}\left(\omega_{13} \wedge \omega_{23}\right) \geq 4 \pi k-4 \pi(\cos \phi)^{2}$. Now, integrating on the northern hemisphere we have

$$
\begin{aligned}
2 \int_{0}^{\pi / 2} \int_{S_{\theta}} i^{*}\left(\omega_{13} \wedge \omega_{23}\right) & \left(e_{1}, \epsilon_{1} \tilde{e}\right) v_{\theta} d \theta \\
& \geq 2 \int_{0}^{\pi / 2}\left(4 \pi k-4 \pi \cos ^{2} \theta\right) d \theta=4 \pi^{2} k-2 \pi^{2}>0
\end{aligned}
$$

Taking absolute values in (11) we obtain

$$
\left|2 \int_{0}^{\pi / 2} \int_{S_{\theta}} i^{*}\left(\omega_{13} \wedge \omega_{23}\right)\left(e_{1}, \epsilon_{1} \tilde{e}\right) v_{\theta} d \theta\right| \geq\left|4 \pi^{2} k-2 \pi^{2}\right| .
$$

For the second integral of (4) we work in a similar way. Now the index of $\vec{v}$ at $S$ is $-k<0$ and the aim is to obtain an inequality equivalent to (11) in such a way that we know the sign of both sides of the inequality.

For $-\pi / 2 \leq \phi<\psi \leq 0$, the inequality $\int_{S_{\phi}} i^{*}\left(\omega_{13} \wedge \omega_{23}\right) \geq \int_{S_{\psi}} i^{*}\left(\omega_{13} \wedge \omega_{23}\right)-$ $\operatorname{vol} S_{\psi}-\operatorname{vol} S_{\phi}$ in (9) still holds. Now, the index of $\vec{v}$ at $S$ is given by

$$
\lim _{\phi \rightarrow-\pi / 2} \int_{S_{\phi}} i^{*}\left(\omega_{13} \wedge \omega_{23}\right)=-4 \pi k .
$$

From this and the inequality in (9) we obtain $-4 \pi k \geq \int_{S_{\psi}} i^{*}\left(\omega_{13} \wedge \omega_{23}\right)-$ $4 \pi(\cos \psi)^{2}$. So, on the southern hemisphere we have

$$
\begin{aligned}
2 \int_{-\pi / 2}^{0} \int_{S_{\theta}} i^{*}\left(\omega_{13} \wedge \omega_{23}\right) & \left(e_{1}, \epsilon_{1} \tilde{e}\right) v_{\theta} d \theta \\
\leq & 2 \int_{-\pi / 2}^{0}\left(-4 \pi k+4 \pi \cos ^{2} \theta\right) d \theta=-4 \pi^{2} k+2 \pi^{2}<0
\end{aligned}
$$


For the absolute value we get the bound

$$
\begin{aligned}
\left|2 \int_{-\pi / 2}^{0} \int_{S_{\theta}} i^{*}\left(\omega_{13} \wedge \omega_{23}\right)\left(e_{1}, \epsilon_{1} \tilde{e}\right) \nu_{\theta} d \theta\right| & \geq\left|-4 \pi^{2} k+2 \pi^{2}\right| \\
& =\left|4 \pi^{2} k-2 \pi^{2}\right| .
\end{aligned}
$$

Finally, the energy of $\vec{v}$ follows from (4), (12) and (14) that is bounded by:

$$
\begin{aligned}
\mathscr{E}(\vec{v}) & \geq \frac{1}{2}\left|4 \pi^{2} k-2 \pi^{2}\right|+\frac{1}{2}\left|4 \pi^{2} k-2 \pi^{2}\right|+\frac{3}{2} \operatorname{vol} \mathbf{S}^{3} \\
& \geq \frac{1}{2}\left|4 \pi^{2} k+4 \pi^{2} k-4 \pi^{2}\right|+\frac{3}{2} \operatorname{vol} \mathbf{S}^{3}=\left(|2 k-1|+\frac{3}{2}\right) \operatorname{vol} \mathbf{S}^{3} .
\end{aligned}
$$

This completes the proof of Theorem 4 .

Remarks. (1) When $\vec{v}=V_{R}$, equality is attained in Theorem 4. This can be shown by direct computation, but also by reproducing the proof with the following considerations: the acceleration of $V_{R}$ is zero, $V_{R}^{\perp}$ is integrable and umbilical, and the angle $\alpha$ is equal to $\pi / 2$.

(2) In a sense, the assumption of Theorem 4 about the quantity and the antipodal position of the singularities is a necessary restriction. It is possible to construct a sequence of unit vector fields, each with an arbitrary number of singularities in free positions, whose energy converges to the energy of the radial vector field $V_{R}$.

\section{Acknowledgements}

We thank Professor Fabiano Brito from the University of São Paulo (Brazil) for encouraging us to tackle this line of investigations.

\section{References}

[Berndt et al. 2003] J. Berndt, L. Vanhecke, and L. Verhóczki, "Harmonic and minimal unit vector fields on Riemannian symmetric spaces", Illinois J. Math. 47 (2003), 1273-1286. MR 2005i:53064 Zbl 1045.53036

[Boeckx et al. 2002] E. Boeckx, J. C. González-Dávila, and L. Vanhecke, "Stability of the geodesic flow for the energy", Comment. Math. Univ. Carolin. 43:2 (2002), 201-213. MR 2003g:53147 Zbl 02185229

[Borrelli et al. 2003] V. Borrelli, F. Brito, and O. Gil-Medrano, "The infimum of the energy of unit vector fields on odd-dimensional spheres”, Ann. Global Anal. Geom. 23:2 (2003), 129-140. MR 2003m:53046 Zbl 1031.53090

[Brito 2000] F. G. B. Brito, "Total bending of flows with mean curvature correction", Differential Geom. Appl. 12:2 (2000), 157-163. MR 2001g:53065 Zbl 0995.53023

[Brito and Salvai 2004] F. Brito and M. Salvai, "Solenoidal unit vector fields with minimum energy", Osaka J. Math. 41:3 (2004), 533-544. MR 2005h:53044 Zbl 1076.53078

[Brito and Walczak 2000] F. G. B. Brito and P. Walczak, "On the energy of unit vector fields with isolated singularities”, Ann. Polon. Math. 73:3 (2000), 269-274. MR 2001k:53049 Zbl 0997.53025 
[Chacón and Naveira 2004] P. M. Chacón and A. M. Naveira, "Corrected energy of distributions on Riemannian manifolds”, Osaka J. Math. 41:1 (2004), 97-105. MR 2004k:53043 Zbl 1055.53021

[Gil-Medrano and Hurtado 2004] O. Gil-Medrano and A. Hurtado, "Spacelike energy of timelike unit vector fields on a Lorentzian manifold”, J. Geom. Phys. 51:1 (2004), 82-100. MR 2005f:53115 Zbl 1076.53086

[Gil-Medrano and Llinares-Fuster 2001] O. Gil-Medrano and E. Llinares-Fuster, "Second variation of volume and energy of vector fields. Stability of Hopf vector fields", Math. Ann. 320:3 (2001), 531-545. MR 2002m:53100 Zbl 0989.53020

[Gil-Medrano et al. 2004] O. Gil-Medrano, J. C. González-Dávila, and L. Vanhecke, "Harmonicity and minimality of oriented distributions", Israel J. Math. 143 (2004), 253-279. MR 2005g:58004 Zbl 1073.58007

[González-Dávila and Vanhecke 2002] J. C. González-Dávila and L. Vanhecke, "Energy and volume of unit vector fields on three-dimensional Riemannian manifolds", Differential Geom. Appl. 16:3 (2002), 225-244. MR 2003b:53032 Zbl 1035.53089

[Wiegmink 1995] G. Wiegmink, "Total bending of vector fields on Riemannian manifolds", Math. Ann. 303:2 (1995), 325-344. MR 97a:53050 Zbl 0834.53034

[Wiegmink 1996] G. Wiegmink, "Total bending of vector fields on the sphere $S^{3 \text { ", Differential }}$ Geom. Appl. 6:3 (1996), 219-236. MR 97e:53067 Zbl 0866.53025

[Wood 2000] C. M. Wood, "The energy of Hopf vector fields", Manuscripta Math. 101:1 (2000), 71-88. MR 2001c:53090 Zbl 0946.53032

Received November 7, 2005. Revised February 2, 2007.

\author{
PABLO M. CHACÓN \\ Departamento de Matemáticas \\ FACUlTAD DE CiENCIAS \\ UNIVERSIDAD DE SALAMANCA \\ Plaza DE LA MERCED 1-4 \\ 37008 SALAMANCA \\ SPAIN \\ pmchacon@usal.es \\ GIOVANNI DA SILVA NUNES \\ Universidade Federal de PElotas \\ Instituto de FísicA E MATEMÁtica \\ CAMPUS UNIVERSitÁRIO - CAPÃo DO LEÃo \\ CAIXa Postal 354 \\ 96010-900 PELOTAS, RS \\ BRAZIL \\ gsnunes@portoweb.com.br
}

